\title{
Diffusion Kurtosis Imaging
}

National Cancer Institute

\section{Source}

National Cancer Institute. Diffusion Kurtosis Imaging. NCI Thesaurus. Code C116487.

An extension of conventional diffusion tension imaging, which estimates the kurtosis of the water diffusion probability distribution function. This technique is most commonly used to study the brain. 